\title{
Seminal transferrin in the seminal quality evaluation of hemodialytic patients
}

\author{
Gilmar Pereira Silva ${ }^{1}$, Fabiana Pirani Carneiro ${ }^{1}$,Vitor Pereira Xavier Grangeiro ${ }^{2}$ \\ ${ }^{1}$ University of Brasilia, Brasilia, Federal District, Brazil; \\ ${ }^{2}$ Faculty of Medical Sciences, João Pessoa, Paraiba, Brazil.
}

\begin{abstract}
Summary Objective: to verify the association between seminal quality and seminal transferrin (ST)

level and fertility index in patients undergoing chronic hemodialysis $(\mathrm{CH})$.

Material and methods: This is a cross-sectional study in a group of 60 men (case) undergoing $\mathrm{CH}$ for more than 6 months, and a group of 30 healthy men (control), aged 18-60 years, without clinical or laboratory signs of infection/inflammation. Spermiogram was performed, fertility index (FI) was calculated and ST and sex hormones (SH) levels were measured, including follicle-stimulating hormone, luteinizing hormone, total testosterone, and prolactin.

Results: All individuals were eugonadal. No differences for age $(49.47 \pm 5.56,47.90 \pm 6.2, p=0.22)$ were observed between cases and controls, whereas there were significant differences between the individuals in the case and control groups with respect to the mean FI $(p=0.000)$, seminal parameters $(S P)$ $(p=0.000)$, and ST levels $(40.12 \pm 08.25$ vs $73.32 \pm 06.8$, $p=0.000)$. ST levels were correlated with FI $(r=0.787$, $p=0.00)$ and SP (motility: $r=0.857, p=0.000$; vitality: $r=0.551, p=0.000$; density: $r=0.850, p=0.000$; normal morphology: $r=0.386, p=0.000$ ). Linear regression model showed relationship of ST levels with total sperm motility $\left(R^{2}=0.701 ; p=0.000\right)$ and and FI $\left(R^{2}=0.569 ; p=0.000\right)$. Conclusions: Our results suggest that seminal quality is associated with ST levels and FI and that it can be used the initial investigation of subfertility/infertility of patients undergoing chronic hemodialysis..
\end{abstract}

KEY WORDS: Chronic kidney disease; Hemodialysis; Male infertility; Seminal transferrin; Seminal quality; Seminal parameters.

Submitted 8 January 2018; 20 February 2018

\section{MATERIALS AND METHODS}

\section{Recruitment, inclusion and exclusion}

A cross-sectional study was realized in the Hemodialysis Sector of the University Hospital of the University of Brasilia, between July 2016 and December 2016, after approval by the Research Ethics Committee of the Faculty of Health Sciences of the University of Brasilia under number 53172316.9.0000.0030.

Inclusion criteria were: age between 18 to 60 years, has been in hemodialysis (HD) for more than 6 months (cases) and absence of acute or chronic liver disease. Exclusion criteria were the presence of hemochromatosis or diseases of iron metabolism.
Patients with hypogonadism and clinical conditions that could alter ST levels such as recent history of genitourinary tract infection, clinical signs of acute or chronic infection/inflammation, positive serology for hepatitis B, $C$ and human immunodeficiency virus (HIV), vascular access infection, leukocytosis, fever, hypoproteinemia were not included in the study. Sample consisted of 60 men (cases) in high flow HD by vascular fistula access, $3 \mathrm{x}$ week with duration of 4 hours/HD session and 30 healthy men (control) from the health promotion outpatient clinic of the same hospital without injury of renal function (glomerular filtration rate $\geq$ than $90 \mathrm{ml} / \mathrm{min}$ per $1.73 \mathrm{~m}^{2}$ ) and sperm without changes.

Routine collection of blood and semen

The blood sample for analysis was collected from the arteriovenous fistula immediately before the first weekly hemodialysis session in the case group and on a previously scheduled day for the control group, always between 8:00 and 10:00 a.m. in the clinical laboratory of the same hospital to assay ST, follicle stimulating hormone (FSH); luteinizing hormone ( $\mathrm{LH}$ ) and total testosterone (TT). On the same day of blood collection, the semen was collected by voluntary masturbation in an appropriate environment to perform spermiogram by manual method according to the guidelines of the World Health Organization (WHO) laboratory manual for the examination and processing of human semen $5^{\text {th }}$ ed (1). The seminal plasma was prepared by centrifuging at $3500 \times$ $\mathrm{g}$ for $20 \mathrm{~min}$ after 30 minutes liquefaction. The supernatant was collected into a new tube and held at $-20^{\circ} \mathrm{C}$ for the measurement of ST levels.

ST and hormones were measured by enzyme immunochemiluminescence using the Immulite 2000/Siemens automatic analyzer. Specific kits were used for quantification, as well as calibrators and controls recommended by the manufacturer.

\section{Fertility Index (FI)}

FI was calculated according to Harvey (2) as follows: FI = sperm concentration $\left(\mathrm{x} 10^{6} / \mathrm{ml}\right) \times$ sperm motility $\mathrm{x}$ percentage of spermatozoa with normal morphology.

\section{Statistical analysis}

After the normal distribution curve of the sample was verified by normality tests (Shapiro-Wilk), for differences 
Table 1.

Comparative evaluation of age, semen and hormone parameters, fertility index and transferrin seminal levels among case and control groups ( $\pm \pm s d)$.

\begin{tabular}{|lccc|}
\hline Observed parameters & Case $\mathbf{n}(\mathbf{6 0})$ & Control $\mathbf{n}(\mathbf{3 0})$ & P values \\
\hline Age (y) & $49,47 \pm 5,56$ & $47,90 \pm 6,22$ & 0,229 \\
\hline Semen volume (ml) & $01,33 \pm 00,36$ & $02,77 \pm 0,44$ & 0,000 \\
\hline Total motility (PR + NP \%) & $34,00 \pm 06,24$ & $71,31 \pm 7,86$ & 0,000 \\
\hline Sperm vitability (\%) & $47,49 \pm 07,31$ & $64,41 \pm 2,89$ & 0,000 \\
\hline Sperm density (x 106/ ml) & $14,95 \pm 06,18$ & $50,21 \pm 8,57$ & 0,000 \\
\hline Morphology sperm (\%) & $25,40 \pm 07,87$ & $59,76 \pm 10,58$ & 0,000 \\
\hline FSH (mlU/ml) & $06,30 \pm 01,21$ & $03,40 \pm 00,48$ & 0,000 \\
\hline LH (mlU/ml) & $15,91 \pm 02,62$ & $02,84 \pm 00,54$ & 0,000 \\
\hline Testosterone (ng/ml) & $04,11 \pm 00,58$ & $05,10 \pm 00,92$ & 0,000 \\
\hline Prolactin (ng/ml) & $16,38 \pm 02,92$ & $05,86 \pm 01,93$ & 0,000 \\
\hline Fertility index & 0,85 (0,57) & $5,54(1,3)$ & 0,000 \\
\hline Seminal transferrin(ng/ml) & $40,12 \pm 08,25$ & $73,32 \pm 06,81$ & 0,000 \\
\hline PR - Progressive motility; NP - non-progressive motility; FSH - follicle-stimulating hormone; & \\
LH - luteinizing hormone; a- $t$ tests. & & & \\
\hline
\end{tabular}

Table 2.

Correlational evaluation Pearson' between seminal transferrin and fertility index and seminal parameters in case group.

\begin{tabular}{|llcc|}
\hline Observed parameters & & \multicolumn{2}{c|}{ Case $\mathbf{n}(\mathbf{6 0})$} \\
& & r & p \\
\hline \multirow{2}{*}{ Seminal } & Fertility index & 0,787 & 0,000 \\
transferrin & Sperm motility & 0,857 & 0,000 \\
versus & Sperm viability & 0,551 & 0,000 \\
& Sperm density & 0,850 & 0,000 \\
& Sperm morphology & 0,386 & 0,002 \\
\hline
\end{tabular}

between two independent quantitative variables was used t-test and Pearson correlation analysis. Statistical significance was set at $\mathrm{p}<0.05$ to reject the null hypothesis. SPSS ${ }^{\circledR}$ for Windows, version 24.0 was used.

\section{RESULTS}

All sample of patients were eugonadics (normal LH, FSH, TT). No difference for afe was observed between cases and controls $(49.47 \pm 5.56,47.90 \pm 6.2, p=$ $0.229)$, whereas significant differences between case and control means were observed for FI ( $p=0.000)$, seminal parameters (SPs) $(\mathrm{P}=0.000)$ and ST levels $(40.12 \pm$ 08.25 vs $73.32 \pm 06.8, \mathrm{p}=0.000)$. ST maintained correlation (Table 2) with FI $(r=0.787, p=0.00)$ and SPs (motility $\mathrm{r}=0.857$ and $\mathrm{p}=0.000$; vitality $\mathrm{r}=0.551$ and $\mathrm{p}=0.000$; density $\mathrm{r}=0.850$ and $\mathrm{p}=0.000$ and normal morphology $r=0.386$ and $p=0.000)$ and without hormones corrections $(p>0,05)$. The positive relationships between ST and sperm concentration and fertility index in group case can be explained by the linear regression model in up to $72.2 \%$ and $56.9 \%$, respectively.

\section{Discussion}

This study is of great importance because it is the first to verify the association between ST levels and SP and FI in patients undergoing HD. The practicality and low cost of the ST measurement can be attractive for the initial evaluation of semen quality in patients undergoing HD with suspected subfertility/infertility.

ST is an isoform of the human transferrin family that is found abundantly in human seminal plasma; it is produced and secreted (80\%) by Sertoli cells (3). It is sensitive to systemic and site inflammatory changes, and is involved in transport of iron ions in the systemic or site compartments (4). Distribution of the mean values of the parameters evaluated in case and control groups can be observed in Figure 1.

The similar age $(p=0.229)$ and eugonadal status $(p=0.000)$ (Table 1$)$ of the studied individuals ensures greater reliability, and it lowers the impact on the analysis and interpretation of these variables.

The male hormonal profile most commonly observed in patients in HD includes elevated serum levels of FSH, LH, and decreased levels of TT in response to the inhibitory action of one or more hypothalamic-pituitary-gonadal axis sites (5). These changes are promoted by factors such as uremia, oxidative stress (OS) and pro-inflammatory cytokines present in HD (4). The hormones profile (Table 1) in the case group in our study followed partially the pattern described above and found by other Authors $(6,7)$ that is high levels of FSH and LH although TT levels were within the limits of normality (eugonadics). Absence of clinical hypogonadism, in thesis, minimizes the effect of hormonal factor in the pathophysiology of the changes found in the ST level and seminal parameter. In the present study there was no relationship $(\mathrm{P}>0.05)$ between ST levels and hormones (Table 2) which is corroborated by studies of Fuse (8) and Ber (9). However, there are studies that show as Tfs was inversely correlated with serum levels of LH, FSH and prolactin $(10,11)$.

ST levels found were significantly lower in the cases in relation to control in agreement with previous studies conducted by different researchers in non-uremic populations with suspected sub/infertility. Kosar et al. (12) found values of $58.1 \pm 14.4 \mu \mathrm{g} / \mathrm{ml}$ vs $108.4 \pm 17.5 \mu \mathrm{g} / \mathrm{ml}$ $(\mathrm{p}<0.0001)$; Bharshankar and Bharshankar (13) $2.63 \pm$ $1.76 \mathrm{mg} / \mathrm{dl}$ vs $5.35 \pm 2.07 \mathrm{mg} / \mathrm{dl}(\mathrm{p}<0.001)$ and Saeed et al. (14) $54.0 \mu \mathrm{g} / \mathrm{ml}(50.0-60,0)$ vs $74.0 \mu \mathrm{g} / \mathrm{ml}(69.0-$ 80.0) $(\mathrm{p}<0.01)$.

The explanation of the decrease in mean ST levels in unhealthy men in sub/infertility studies are not well known, but it is hypothesized that it is due to the action of interleukin-6 (IL6) in testis to reduces transferrin secretion in Sertoli cells (15).

All sperm cell parameters evaluated (Table 1) were significantly lower in the case group than in the control group ( $p=0.000)$, corroborating partially the results of other authors $(16,17)$ that did not found significant differences for all the elements of the SP $(16,17)$. However there is a study that did not show a significant difference in ST levels between healthy subjects and those with seminal quality alterations (18). There was a statistically significant correlation (Table 2) between ST and all ele- 


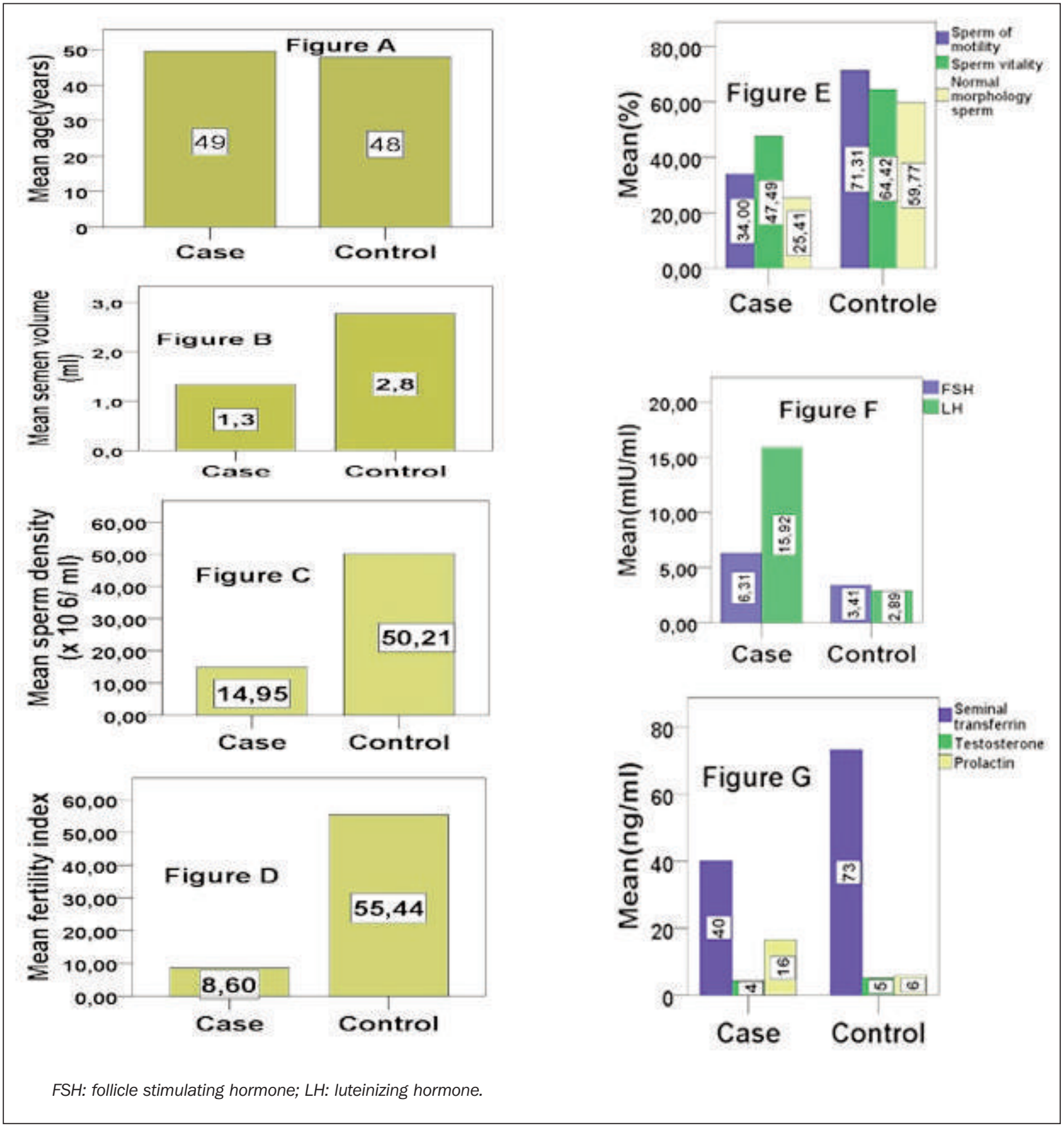

Figure 1.

Distribution of mean values of all parameters tested in the group and control group.

ments of the SP $(p<0.05)$. However, these correlations were not linear in comparative studies with non-uremic population of other authors. Bharshankar and Bharshankar (13) found only a statistically significant correlation between ST and percent of motile sperms $(\mathrm{p}<0.05)$.

Fuse et al. (8) found only a statistically significant correlation between sperm concentration and ST level $(r=0.56$; $\mathrm{p}<0.05)$.

The FI is a general index to evaluate the semen quality, which was better than any single semen parameter (2).

In the present study we found a FI significantly lower in the case group than in the control group (Table 1) in according with the results of other Authors in uremic populations as Xu et al. (16) who found a value of 0.68 (2.08) vs $7.72(13.51)(\mathrm{p}<0.05)$ and $X u$ et al. $(17)$ who reported a value of $0.23(0.76)$ vs $13.02(14.26)(\mathrm{p}<0.001)$.

The positive relationship of ST with sperm concentration and fertility index in the present study can explain up to $72.2 \%\left(R^{2}\right)(p=0.000)$ and $61.9 \%\left(R^{2}\right)(p=0.000)$ of the cases, respectively, by the simple linear regression model. This reinforces the hypothesis of its association with SPs. The explanation for changes identified in SPa and ST level are multifactorial (19). But there is evidence of the importance of cytokines and other immune regulatory factors in 
the regulation of testicular function (steroidogenesis and spermatogenesis) during pathophysiological states as well as under normal physiological conditions (20).

Cytokines are interconnected with multiple factors, including steroid hormones, the redox system, and systemic or local inflammation (15).

The HD patients are characterized by increased levels of oxidative stress and inflammation with the relationship between inflammation and oxidative stress leading to overproduction of reactive oxygen species (ROS) (21).

These factors are likely to represent an important component for the development of SP changes (21).

It is postulated that hypercytokinemia generated in patient HD can profoundly affect vascular testicular permeability and to achieve interstitial compartment of the testis (22). This causes directly profound changes in the physiology of the blood-testis barrier (BTB) and/or stimulate the testicular macrophages to produce differents pro and anti-inflammatory cytokines, promoting blockage of the paracrine/autocrine testicular regulation systems (22). In the male reproductive system there is a variety of cytokines in human seminal plasma, with differences in cytokine concentrations between fertile and infertile men and negative correlations between some cytokine levels and SP (22).

The relationship between inflammation and oxidative stress is confirmed and both processes contribute with adverse effects on the structural and functional integrity of sperm, resulting in changes in the sperm function and in male infertility by protein, glycogen, lipid and DNA peroxidation that can partially justify changes in the seminal parameters (15).

Pro- and anti-inflammatory cytokines and other inflammatory mediators are largely responsible for the changes observed in seminal plasma (23). IL-6 is a multifunctional cytokine involved in many changes identified in the tubular and interstitial compartments testicular with reflection on the seminal quality (23). It has been suggested that it is a potent inhibitor of the seminiferous epithelium (23), modulating ST production by Sertoli cells (20) and inducing persistent testicular resistence to LH action and/or suppression of cell steroidogenesis in the Leydig cells (24),

On the other hand, IL-6 has other important functions which play an important role in maintaining the function of Sertoli cells, germ cells, regulating the dynamics of BTB via delaying BTB-constituent proteins degradation (22). Overexpression of IL-6 (systemic inflammation) could disrupt the integrity of the Sertoli cell and BTB, making foreign molecules to reach germ cells (22). Alteration of the permeability of BTB seems to be the most important event in the pathophysiology of changes in the semen of patients with seminal parameter changes (22). The cytokines produced by the complex systemic inflammation/oxidative stress, frequently observed in these patients might modulate the activity of the prooxidative and antioxidative systems (25).

The oxidative stress is responsible for permanent peroxidative damage to spermatozoa by means of protein complexes called ion-responsive elements and ion regulatory proteins (ERIs/PRIs) (25). These proteins are responsible for the post-transcriptional regulation of proteins linked to uptake iron (transferrin receptor 1 -RTfl) and storage iron (ferritin) (25). When altered, they promote changes in intracellular iron ion levels and morpho-functional changes in seminal quality by toxic effect on germ cells (25). That breakdown of the intratesticular pro-and antiinflammatory cytokine balance promoted by different cytokines as the IL- 6 contributes significantly by changes in the permeability of BTB to important alterations in autocrine/paracrine autoregulation mechanisms of interaction between surrounding germ cells, Sertoli and Leydig cells (26). The changes promoted by cytokines result in an immunopathological microenvironment that can change the testicular immune privilege and justify partially the changes in ST levels and their association with seminal quality and fertility index in this group of patients.

\section{ConcLusion}

Although the multifactoriality in etiology of sub/infertility, our results suggest that seminal quality is associated with ST levels and what ST can be used the initial investigation of subfertility/infertility of patients undergoing chronic hemodialysis with alteration in seminal quality. This study presents the limitations of the absence of supplementary measurement of total seminal antioxidant capacity and of the reduced sample.

\section{REFERENCES}

1. Organization WH. WHO laboratory manual for the examination and processing of human semen. 2010.

2. Harvey C. A fertility index derived from semen analysis. J Clin Pathol. 1953; 6:232-6.

3. Franca LR, Hess RA, Dufour JM, et al. The Sertoli cell: one hundred fifty years of beauty and plasticity. Andrology. 2016; 4:189212

4. Akchurin OM, Kaskel F. Update on inflammation in chronic kidney disease. Blood Purif. 2015; 39:84-92.

5. Palmer BF, Clegg DJ. Gonadal dysfunction in chronic kidney disease. Rev Endocr Metab Disord. 2017; 18:117-130.

6. Zedan H, Kamal EE, El Shazly A, et al. Impact of renal failure and haemodialysis on semen parameters and reproductive hormones. Human Andrology. 2013; 3:16-20.

7. Lehtihet M, Hylander B. Semen quality in men with chronic kidney disease and its correlation with chronic kidney disease stages. Andrologia. 2015; 47:1103-8.

8. Fuse H, Satomi S, Okumura M, Katayama T. Seminal plasma transferrin concentration: relationship with seminal parameters and plasma hormone levels. Urol Int. 1992; 49:158-62.

9. Ber A, Vardinon N, Yogev L, et al. Transferrin in seminal plasma and in serum of men: its correlation with sperm quality and hormonal status. Hum Reprod. 1990; 5:294-7.

10. Irisawa C, Nakada T, Kubota Y, et al. Transferrin concentration in seminal plasma with special reference to serum hormone levels in infertile men. Arch Androl. 1993; 30:13-21.

11. Meeker JD, Godfrey-Bailey L, Hauser R. Relationships between serum hormone levels and semen quality among men from an infertility clinic. J Androl. 2007; 28:397-406. 
12. Kosar A, Sarica K, Ozdiler E. Effect of varicocelectomy on seminal plasma transferrin values: a comparative clinical trial. Andrologia. 2000; 32:19-22.

13. Bharshankar RN, Bharshankar JR. Relationship of seminal plasma transferrin with seminal parameters in male infertility. Indian J Physiol Pharmacol. 2000; 44:456-60.

14. Saeed S, Khan FA, Rahman SB, et al. Biochemical parameters in evaluation of oligospermia. J Pak Med Assoc. 1994; 44:137-40.

15. Fraczek M, Kurpisz M. Cytokines in the male reproductive tract and their role in infertility disorders. J Reprod Immunol. 2015; 108:98-104.

16. Xu L, Xu H, Zhu X, et al. Effect of uremia on semen quality and reproductive function in humans. Cell Biochem Biophys. 2012; 62:29-33.

17. Xu LG, Xu HM, Zhu XF, et al. Examination of the semen quality of patients with uraemia and renal transplant recipients in comparison with a control group. Andrologia. 2009; 41:235-40.

18. Eneroth P, Lizana J, Bygdeman M. Lactoferrin and transferrin levels in the seminal plasma of infertile men. Protides of the Biological Fluids. 1984; 31:149-53.

19. Coutton C, Fissore RA, Palermo GD, et al. Male Infertility:
Genetics, Mechanism, and Therapies. Biomed Res Int. 2016; 2016:7372362.

20. Guazzone VA, Jacobo P, Theas MS, Lustig L. Cytokines and chemokines in testicular inflammation: A brief review. Microsc Res Tech. 2009; 72:620-8.

21. Tucker PS, Scanlan AT, Dalbo VJ. Chronic kidney disease influences multiple systems: describing the relationship between oxidative stress, inflammation, kidney damage, and concomitant disease. Oxid Med Cell Longev. 2015; 2015:806358.

22. Zhang $H$, Yin $Y$, Wang $G$, et al. Interleukin-6 disrupts bloodtestis barrier through inhibiting protein degradation or activating phosphorylated ERK in Sertoli cells. Sci Rep. 2014; 4:4260

23. Salman DTA-WD. Evaluation the effect of Interleukin-6 and Tumor Necrosis Factor in semen quality of infertile men with varicocele. Kufa Journal for Nursing Sciences | مولعل | 2016; 6.

24. Tremblay JJ. Molecular regulation of steroidogenesis in endocrine Leydig cells. Steroids. 2015; 103:3-10.

25. Zhao S, Zhu W, Xue S, Han D. Testicular defense systems: immune privilege and innate immunity. Cell Mol Immunol. 2014; 11:428-37.

26. Fijak M, Bhushan S, Meinhardt A. The Immune Privilege of the Testis. Immune Infertility: Springer; 2017; p. 97-107.

\section{Correspondence}

Gilmar Pereira Silva (Corresponding Author)

gilpsilva2006@gmail.com

Urologist physician $\mathrm{PhD}$, University of Brasilia

Hotel Sector North, Block D, Apartment 1716, Fusion Building,

70701- 040 Brasilia, Federal District, Brazil

Fabiana Pirani Carneiro

fabianapirani@hotmail.com

Professor PhD, Faculty of Medicine of the University of Brasilia

Brasilia, Federal District, Brazil

Vitor Pereira Xavier Grangeiro

vitorpxavierg10@gmail.com

Academic of the Faculty of Medical Sciences

João Pessoa, Paraiba, Brazil 